\title{
Total Angular Momentum Dichroism of the Terahertz Vortex Beams at the Antiferromagnetic Resonances
}

\author{
A. A. Sirenko $\odot,{ }^{1,2,}{ }^{*}$ P. Marsik, ${ }^{2}$ L. Bugnon, ${ }^{2}$ M. Soulier, ${ }^{2}$ C. Bernhard, ${ }^{2}$ T. N. Stanislavchuk, ${ }^{1}$ \\ Xianghan $\mathrm{Xu}^{3}{ }^{3}$ and S.-W. Cheong ${ }^{3}$ \\ ${ }^{1}$ Department of Physics, New Jersey Institute of Technology, Newark, New Jersey 07102, USA \\ ${ }^{2}$ Department of Physics, University of Fribourg, CH-1700 Fribourg, Switzerland \\ ${ }^{3}$ Rutgers Center for Emergent Materials and Department of Physics and Astronomy, Rutgers University, \\ Piscataway, New Jersey 08854, USA
}

(Received 6 March 2020; revised 5 February 2021; accepted 11 March 2021; published 12 April 2021)

\begin{abstract}
Terahertz vortex beams with different superposition of the orbital angular momentum $l= \pm 1, \pm 2, \pm 3$, and \pm 4 and spin angular momentum $\sigma= \pm 1$ were used to study antiferromagnetic (AFM) resonances in $\mathrm{TbFe}_{3}\left(\mathrm{BO}_{3}\right)_{4}$ and $\mathrm{Ni}_{3} \mathrm{TeO}_{6}$ single crystals. In both materials we observed a strong vortex beam dichroism for the AFM resonances that are split in external magnetic field. The magnitude of the vortex dichroism is comparable to that for conventional circular dichroism due to $\sigma$. The selection rules at the AFM resonances are governed by the total angular momentum of the vortex beam: $j=\sigma+l$. In particular, for $l= \pm 2, \pm 3$, and \pm 4 the sign of $l$ is shown to dominate over that for conventional circular polarization $\sigma$.
\end{abstract}

DOI: 10.1103/PhysRevLett.126.157401

Magnetic properties of quantum matter are at the forefront of condensed matter physics. New optical probes are required for successful chiral discrimination in solids and for control and manipulation of quantum effects. By tapping the vorticity of the light beams and thereby adding an additional degree of freedom in a form of the orbital angular momentum (OAM) $l \hbar$ with $l= \pm 1, \pm 2, \pm 3, \ldots$ [1], optics may facilitate future studies of topological phases [2], magnetic materials with noncollinear spins [3,4], and chiral excitations [5]. Vortex beams can simultaneously carry both OAM and conventional circular polarization in the form of the spin angular momentum (SAM) $\sigma \hbar$ with $\sigma= \pm 1$ [6]. Recently, broadband terahertz vortex beams have been implemented for spectroscopy of ferrimagnetic Dy iron garnet [7]. For beams with $l= \pm 1$, it has been demonstrated that the resonant absorption by magnetic modes of $\mathrm{Dy}^{3+}$ depends on both the handedness of the vortex, or sign of $l$, and the direction of the beam propagation with respect to the sample magnetization. These experiments were complemented by theoretical studies of the optical activity of vortex beams [8]. Here we present qualitatively new experiments for the interaction between the spin waves, or magnons, with higher-order vortex beams with OAM of $l= \pm 2, \pm 3, \pm 4$ in combination with a SAM of $\sigma= \pm 1$. Particularly, we will show that the selection rules for absorption of vortex beams at the antiferromagnetic (AFM) resonances in $\mathrm{TbFe}_{3}\left(\mathrm{BO}_{3}\right)_{4}$ and $\mathrm{Ni}_{3} \mathrm{TeO}_{6}$ are determined by the total angular momentum (AM) $j=l+\sigma$ of light. These two rather different AFM materials have been chosen to highlight the universal aspects of the observed dichroic effects for beams with OAM, SAM, and total AM.
The Ni telluride (NTO) with the formula $\mathrm{Ni}_{3} \mathrm{TeO}_{6}$ and the $\mathrm{Tb}$ ferroborate $(\mathrm{Tb} \mathrm{FB})$ with the formula $\mathrm{TbFe}_{3}\left(\mathrm{BO}_{3}\right)_{4}$ have in common that the corresponding spins of the magnetic ions, $\mathrm{Fe}^{3+}$ and $\mathrm{Ni}^{2+}$, order collinearly along the crystalline $c$ axis below $T_{N}(\mathrm{TbFB})=41 \mathrm{~K}[9,10]$ and $T_{N}(\mathrm{NTO})=52 \mathrm{~K}$ [11]. Moreover, they have similar energies of the AFM resonances at $T \ll T_{N}$ of $\hbar \Omega_{M}(\mathrm{NTO})=0.38 \mathrm{THz}$ and $\hbar \Omega_{M}(\mathrm{TbFB})=0.44 \mathrm{THz}$. In both materials $\hbar \Omega_{M}(T)$ decreases when $T \rightarrow T_{N}$. The crystal and magnetic structures of Tb FB and NTO have several distinct features. $\mathrm{Ni}_{3} \mathrm{TeO}_{6}$ has a monopolar and monochiral $R 3$ structure with 10 atoms in a rhombohedral unit cell $[12,13]$. The $\mathrm{Ni}^{2+}$ ions, which nominally have a ${ }^{3} F_{4}$ configuration with $S=1$ and $L=3$, occupy three (i, ii, and iii) nonequivalent positions. Layers of $\mathrm{Ni}_{i}^{2+} \mathrm{O}_{6} / \mathrm{Te}^{6+} \mathrm{O}_{6}$ octahedra alternate along the $c$ axis with $\mathrm{Ni}_{\mathrm{ii}}^{2+} \mathrm{O}_{6} /$ $\mathrm{Ni}_{\text {iii }}^{2+} \mathrm{O}_{6}$ octahedra. The spin configuration of the $\mathrm{Ni}_{i}^{2+}-\mathrm{Ni}_{\mathrm{ii}}^{2+}-\mathrm{N}_{i}^{2+}-\mathrm{Ni}_{\mathrm{ii}}^{2+}$ network is up-up-down-down, while the $\mathrm{Ni}_{\text {iii }}^{2+}$ spins are parallel to the $\mathrm{Ni}_{\text {ii }}^{2+}$ spins in the same layer. More details about the spin and lattice excitations in NTO are given in Ref. [14]. Tb FB, with two magnetic ions $\mathrm{Tb}^{3+}$ and $\mathrm{Fe}^{3+}$, is a multiferroic with a noncentrosymmetric structure at room temperature described by the trigonal space group $R 32\left(D_{3}^{7}\right)$ that incorporates $\mathrm{FeO}_{6}$ octahedra which form spiral chains along the $c$ axis [15]. At $T=200 \mathrm{~K}$, a structural phase transition takes place into a trigonal $P 3_{1} 21$ phase [16]. Above $4 \mathrm{~K}$, the AFM properties of Tb FB are governed by the moments of the $\mathrm{Fe}^{3+}$ ions in the ${ }^{6} S_{5 / 2}$ configuration with $S=5 / 2$ and $L=0$. The $\mathrm{Tb}^{3+}$ moments order only below $4 \mathrm{~K}$ and remain paramagnetic in all our experiments, albeit they are partially polarized via the $f$ - $d$ exchange interaction with the 
Fe moments [9,10,17]. The optical anisotropy and magnon spectra of Tb FB are described in Refs. [18,19]. The phonons and crystal field transitions in ferroborates are discussed in Refs. [20,21].

The time-domain terahertz optical setup comprises photoconductive antennas as emitter and detector of coherent femtosecond pulses, a mirror optics with $f$ number 10, a liquid He flow optical cryostat, wire-grid linear polarizers, and an optical retarder [7,22]. External magnetic fields $B$ of $\pm 0.47 \mathrm{~T}$ were applied to the samples in the Faraday configuration using permanent ring magnets. The positive magnetic field $\vec{B}$ is parallel with the wave vector $\vec{k} \| \vec{z}$. The time-resolved signal was Fourier transformed to obtain a continuous intensity spectrum $I(\nu)$ with frequencies $\nu$ between 0.1 and $1.65 \mathrm{THz}$. A single Fresnel prism made of cyclic olefin copolymer converted the incident linear polarization $\left(\vec{e}_{x} \pm \vec{e}_{y}\right)$ to the right-hand $\vec{e}_{R}=\vec{e}_{x}-i \cdot \vec{e}_{y}$ or left-hand $\vec{e}_{L}=\vec{e}_{x}+i \cdot \vec{e}_{y}$ circular polarizations. An axicon retarder made by TYDEX [23] was used to produce broadband terahertz vortex beams with OAM $l=+1$ or $l=-1$ and with the electric field distribution of $\vec{e}_{l}(\vec{r}, \phi) \approx$ $(\vec{r} / r) \exp \left[i \cdot l \cdot\left(\phi-\phi_{0}\right)\right]$, where $\phi$ is the vortex beam phase, the initial phase is $\phi_{0}=3 \pi / 4$, and $\vec{r}$ is the radial coordinate [7]. To obtain an OAM with $|l|>1$, the broadband axicon was replaced with pairs of two identical transparent spiral plates that produced the LaguerreGaussian (LG) beams with the OAM at the sample with $\vec{e}_{l}(\phi) \approx \vec{e}_{y} \exp [i \cdot l \cdot \phi]$, where $l$ is integer $l= \pm 2$, \pm 3 , or \pm 4 in the vicinity of the AFM resonances. The first spiral plate created the vortex beam at the sample, while the second plate unwound the vortex wave front at the detector. The handedness of the 3D printed spirals determines the sign of $l$. Several frequencies $\nu_{l}$ with integer $l$ 's were simultaneously presented in the spectra $I(\nu)$ between 0.1 and $1.65 \mathrm{THz}$. The integer value of $l$ at a given frequency $\nu_{l}$ (or wave length $\lambda_{l}$ ) is determined by the step height $h$ and the refractive index $n=1.56$ of the spiral plate: $l \cdot \lambda_{l}=$ $h(n-1)$ [24]. The spiral plates were produced with a Formlabs Form2 3D printer using clear V4 resin, similar to that in Ref. [25]. In addition, terahertz beams with a combined total AM of $j=l+\sigma$ were generated by transmitting circularly polarized beams with $\sigma= \pm 1$ through the same spiral plates producing LG beams with $\vec{e}_{j}(\phi) \approx \vec{e}_{L, R} \exp [i \cdot l \cdot \phi]$. See Supplemental Material for more details of the vortex beam characterization [26].

Figure 1 compares the magnetic dichroism for circularly polarized light with $\sigma= \pm 1$ and for vortex beams with $l=$ \pm 1 at the AFM resonances of NTO at $T=10 \mathrm{~K}$ and for $B \| c$ and shows that they have a similar magnitude and the same sign. As expected [27,28], the magnon peak splits into two modes at $\hbar \Omega_{M}^{ \pm}(B)=\hbar \Omega_{M}(0) \pm \frac{1}{2} g_{\mathrm{Me}} \mu_{B} B$, where $\mu_{B}$ is the Bohr magneton and $g_{\mathrm{Me}}$ is the $g$ factor of the magnetic ion. For NTO we measured $g_{\mathrm{Ni}}=4.1 \pm 0.04$, and Ref. [19] reports that $g_{\mathrm{Fe}}$ is close to 4 . Figures 1 (a) and 1(b) show the two modes of the magnon doublet $\hbar \Omega_{M}^{ \pm}(B)$ that
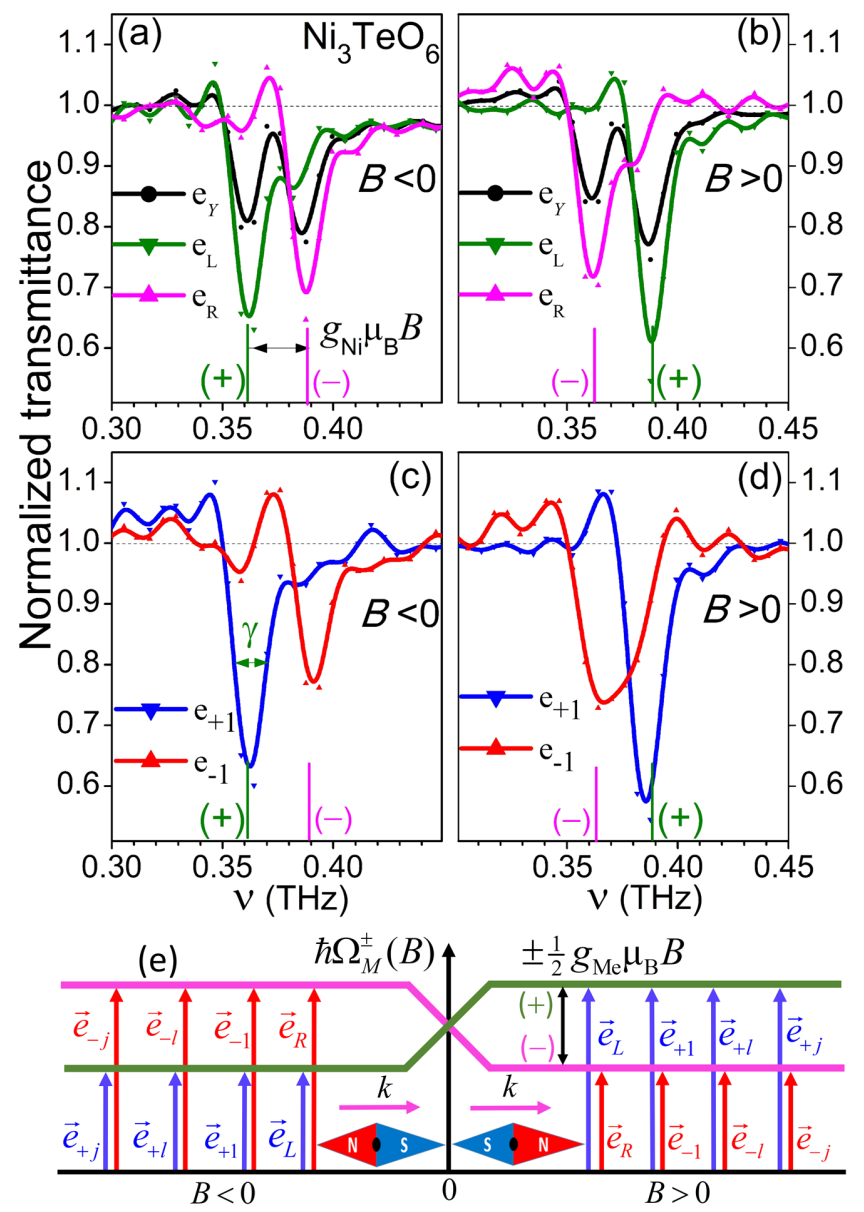

FIG. 1. Magnetic dichroism for circularly polarized light and vortex beams with $l= \pm 1$ in NTO. (a),(b) Normalized transmittance for circularly polarized light, $\vec{e}_{L}$ and $\vec{e}_{R}$, and for conventional linear polarization $\vec{e}_{y}$. (c),(d) Normalized transmittance for broadband vortex beams with $\vec{e}_{+1}$ (blue spectra) and $\vec{e}_{-1}$ (red spectra). All experimental data in (a)-(d) are taken at $B= \pm 0.47 \mathrm{~T}$ and $T=10 \mathrm{~K}$ and are normalized to that measured at $T=60 \mathrm{~K}$ in the same field: $I(\nu, T) / I(\nu, T=60 \mathrm{~K})$. (e) Schematics of the experimentally determined selection rules for the AFM resonances using both circularly polarized light and vortex beams with $l$ and $j$. The positive $\vec{B}$ is parallel with $\vec{k}$.

absorb light of the opposite circular polarization [27] and a reversal of $B$ results in a switch of the selection rules for the sign of the circular polarization. Figures 1(c) and 1(d) show the corresponding absorption spectra for the vortex beams with OAM of $l= \pm 1$ (and no circular polarization) as produced by the axicon retarder [7]. The two modes of the magnon doublet are equally sensitive to the sign of the OAM and the direction of $B$ with respect to $\vec{k}$. A scheme of the circular and vortex dichroisms, which has the same sign for $l, \sigma$, and $j$, is displayed in Fig. 1(e). For both NTO and $\mathrm{Tb} \mathrm{FB}$, the vortex polarization of the magnon peaks is close to $100 \%$ and thus is much larger than the previously reported vortex dichroism of only $\sim 22 \%$ in Dy iron garnet [7]. The strong dichroism in NTO and Tb FB is due to the 


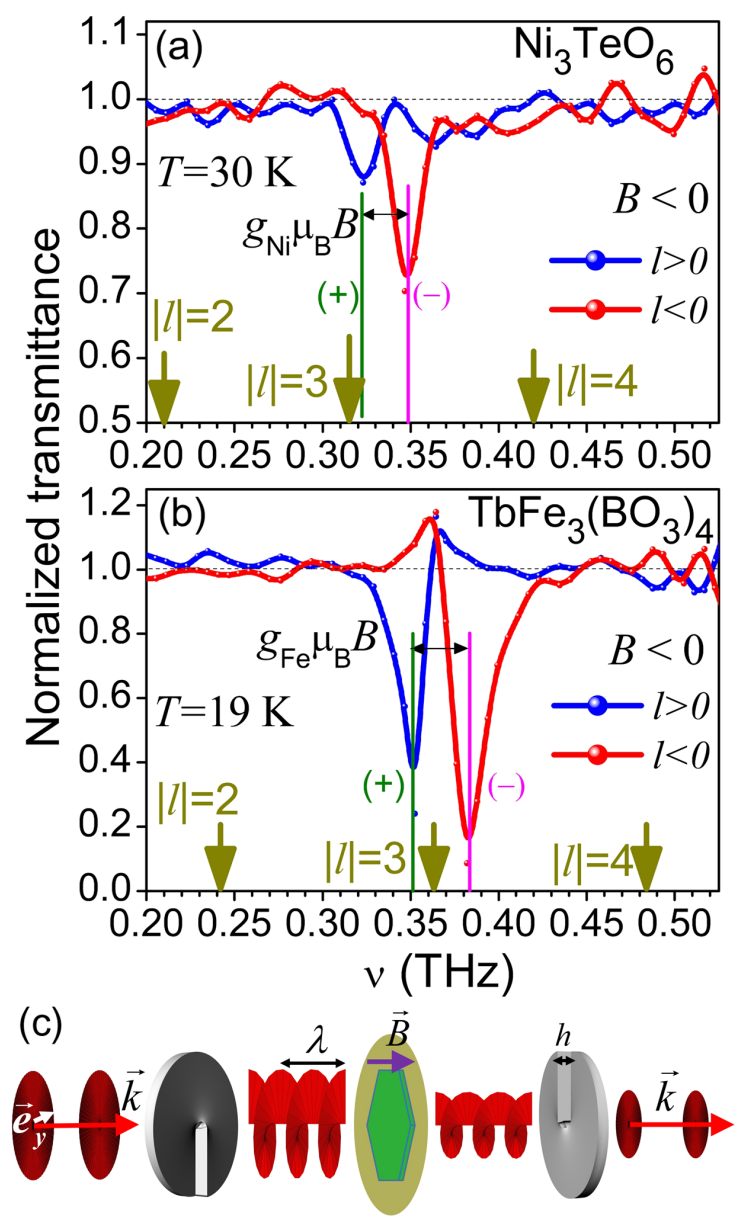

FIG. 2. Vortex dichroism for higher orders of the OAM for (a) NTO at $T=30 \mathrm{~K}$ and (b) Tb FB at $T=19 \mathrm{~K}$. Normalized transmittance spectra for two linearly polarized LG vortex beams $\vec{e}_{l>0}$ (blue spectra) and $\vec{e}_{l<0}$ (red spectra) were taken at $B=$ $-0.47 \mathrm{~T}$ that brings the AFM resonances close to $\nu_{l= \pm 3}$. The corresponding AFM resonances $\hbar \Omega_{M}^{ \pm}(B, T)$ are marked with vertical olive and magenta lines. The frequencies that correspond to $\nu_{l= \pm 2, \pm 3, \pm 4}$ are marked with bold dark yellow arrows. Note the absorption peak asymmetry for the $\vec{e}_{l=+3}$ and $\vec{e}_{l=-3}$ AFM resonances in (b). (c) Schematics for the measurement geometry with linear polarization $\vec{e}_{y}$ for the terahertz beam input. Sample (green hexagon) is placed between a pair of identical spiral plates with step height $h$.

larger splitting of the magnon doublet of $\sim 0.025 \mathrm{THz}$ as compared to the linewidth $\gamma$ of $\sim 0.01 \mathrm{THz}$ of the $\hbar \Omega_{M}^{ \pm}(B)$ branches with $g_{\mathrm{Me}} \mu_{B} B \gg \gamma$.

Next, we discuss the dichroism for the higher-order vortex beams with OAM values of $l= \pm 2, \pm 3$, and \pm 4 so that $\nu_{l= \pm 2,3,4}$ are in proximity with $\hbar \Omega_{M}^{ \pm}(B, T)$ for $T$ between 7 and $30 \mathrm{~K}$. Figures 2(a) and 2(b) show the magnon spectra of NTO and Tb FB for the sample temperatures when $\hbar \Omega_{M}^{ \pm}(B, T) \approx \nu_{l= \pm 3}$. Figure 2(c) shows the experimental configuration. Notably, the higher-order vortex dichroism has the same sign and a similar magnitude as the one described above for $\sigma= \pm 1$ and $l= \pm 1$. An interesting difference between the vortex dichroism experiments with $l= \pm 1$ and $|l| \geq 2$ is as follows. The beams with $l= \pm 1$ [Figs. 1(c) and 1(d)] were produced with an axicon and consisted of a coherent combination of the radial and azimuthal modes [7]. Such a field has a nonzero curl of the electrical field $\vec{e}_{ \pm 1}(\vec{r}, \phi)$ around the beam axis (see Supplemental Material [26]) that is equivalent to a pseudo magnetic field $\vec{B}_{\mathrm{pm}} \approx \nabla \times \vec{e}_{ \pm 1}(\vec{r}, \phi)$ directed along both the $\vec{k}$ vector and the AFM spin $\vec{S}$ ordering axis. The corresponding $\vec{S} \cdot \vec{B}_{\mathrm{pm}}$ interaction may offer an intuitive way of understanding the vortex beam coupling to the ordered spins for experiments shown in Figs. 1(c) and 1(d). In contrast, the LG beams with $|l| \geq 2$ [Figs. 2(a) and 2(b)] have a constant $\vec{e}_{y}$ component across the beam with $\vec{B}_{\mathrm{pm}} \approx$ $\nabla \times \vec{e}_{y}=0$ but a nonzero $\vec{e}_{z}$ due to the vortex phase $\exp [i \cdot l \cdot \phi]$, where $\vec{e}_{z}$ is proportional to $|l|$. The coupling of such LG beams to the AFM spins may be similar to the spin-flip mechanism for intra-Landau-level transitions of 2D electrons in magnetic field [29]. Our experiment thus demonstrates that a coherent vortex phase and $\vec{e}_{z} \cdot \vec{S} \neq 0$ are sufficient to couple a LG vortex beam to the magnetic spin order in matter.

Thus far, we could not identify any strong magnetic modes, other than the magnetic dipoles at $\hbar \Omega_{M}^{ \pm}(B, T)$ that might arise from higher-order spin resonances that are forbidden in the magnetic dipole approximation. However, a possible signature of such higher-order resonances may be attributed to the asymmetry of the AFM modes shown in Fig. 2(b), which might arise from multispin excitations that involve the $\mathrm{Fe}^{3+}$ and $\mathrm{Tb}^{3+}$ spins in $\mathrm{Tb} \mathrm{FB}$ with the extra energy $\hbar \Omega_{\mathrm{Tb}-\mathrm{Fe}}(B, T)$. The shoulder on the low-frequency side in the blue spectrum $(l=+3)$ may correspond to $\hbar \Omega_{M}^{+}(B, T)-\hbar \Omega_{\mathrm{Tb}-\mathrm{Fe}}(B, T)$, while the high-frequency shoulder in the red spectrum $(l=-3)$ may correspond to $\hbar \Omega_{M}^{-}(B, T)+\hbar \Omega_{\mathrm{Tb}-\mathrm{Fe}}(B, T)$. This interpretation is consistent with our findings that the shoulders are the strongest for integer values of $l$ 's at $\hbar \Omega_{M}^{ \pm}(B, T) \approx \nu_{l}$. The shoulders are absent in Fig. 2(a) for NTO which has only $\mathrm{Ni}^{2+}$ magnetic ions.

To further study the interaction mechanism of the optical vortex with magnetism, we produced LG beams with combined orbital $l$ and spin $\sigma$ AM. Figures 3(a) and 3(b) show spectra of $\mathrm{Tb}$ FB measured at $T$ ' $s$ so that $\hbar \Omega_{M}^{ \pm}(B, T) \approx \nu_{l= \pm 3}$ for three $\sigma=0, \pm 1$ and, correspondingly, $j= \pm 2, \pm 3, \pm 4$. These spectra reveal that the AFM resonances coincide for a given $l= \pm 3$ for all three possible $\sigma^{\natural}$ s demonstrating a strong total AM dichroism for which the selection rules of the magnon absorption are determined by the sign of $j$. Note that as long as $|j| \gg|\sigma|$, the AFM resonances are no longer sensitive to the sign of $\sigma$. Figure 4 shows that these selection rules apply not only for the integer values of $j$ at $\hbar \Omega_{M}^{ \pm}(B, T)$ (dark yellow arrows in Figs. 2-4), but in the entire spectral range between $|j|=2$ and $|j|=4$. This suggests that the vortex beam at a 


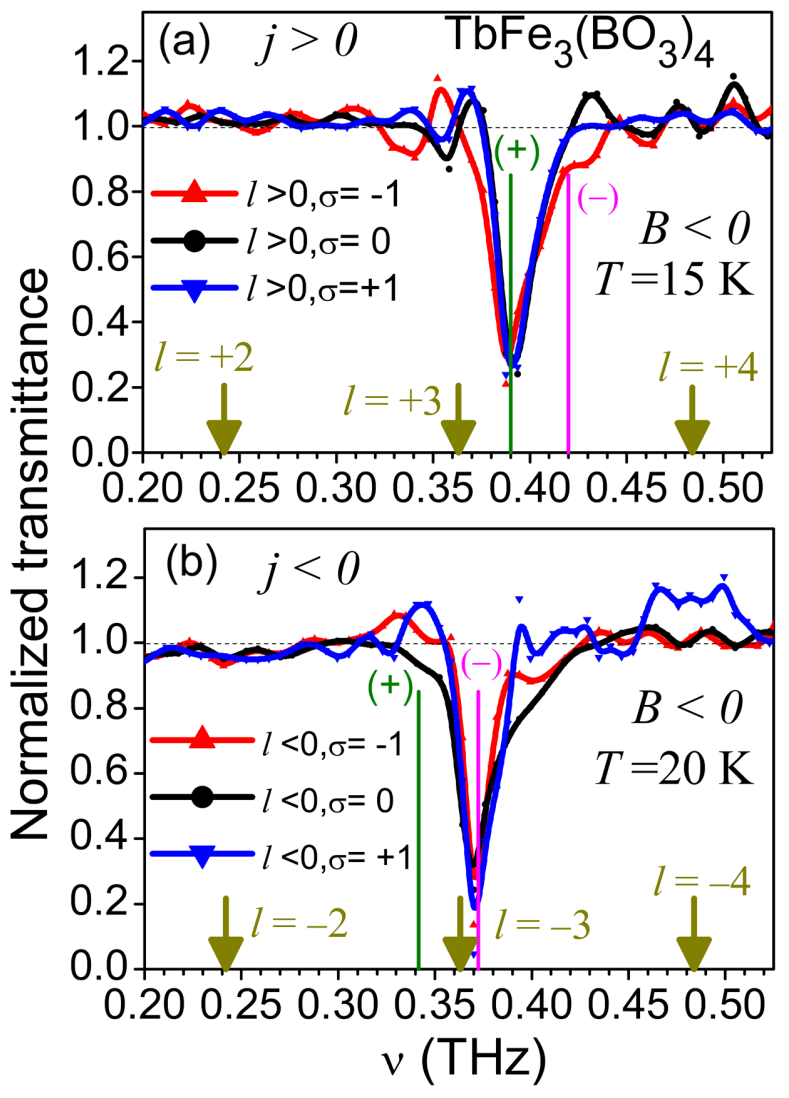

FIG. 3. Normalized transmittance spectra for the LG vortex beams for (a) $j>0$ and (b) $j<0$ in Tb FB at $B=-0.47 \mathrm{~T}$. The resonance frequencies for $\nu_{l= \pm 2}, \nu_{l= \pm 3}$, and $\nu_{l= \pm 4}$ are marked with bold dark yellow arrows. The temperatures were chosen to bring the AFM resonances $\hbar \Omega_{M}^{ \pm}(B, T)$ shown with vertical olive and magenta lines close to $\nu_{l= \pm 3}$ in both (a) and (b). Note that the corresponding AFM resonances coincide for $j=+2(l=+3$, $\sigma=-1), \quad j=+3 \quad(l=+3, \sigma=0), \quad$ and $j=+4 \quad(l=+3$, $\sigma=+1)$ in (a) and for $j=-2(l=-3, \sigma=+1), j=-3$ $(l=-3, \sigma=0)$, and $j=-4(l=-3, \sigma=-1)$ in (b). The measurement geometry is the same as in Fig. 2(c) but with various input polarizations $\vec{e}_{y}$ for black, $\vec{e}_{L}$ for blue, and $\vec{e}_{R}$ for red spectra.

noninteger multiple of the wavelength with respect to $h$, i.e., between $j$ and $j+1$, behaves as a coherent superposition of the beams with $j$ and $j+1$. Another interesting aspect concerns the conservation of the AM when light with a large total AM, e.g., with $|j|=3$, is absorbed by an AFM resonance with a magnetic dipole of $\Delta m_{S}= \pm 1$. This requires that the excess $\mathrm{AM}$ is transmitted to the crystal lattice, e.g., via the interaction with acoustic phonons.

The total AM dichroism in two AFM materials, NTO and $\mathrm{Tb} \mathrm{FB}$, demonstrates that vortex beams with high orders of $l$ can effectively couple to magnetic dipole excitations. The selection rules for the AFM resonances are dictated by the sign of $j$ that dominates over that for conventional circular polarization given by $\sigma$. The different spin and orbital quantum numbers for the ground states of

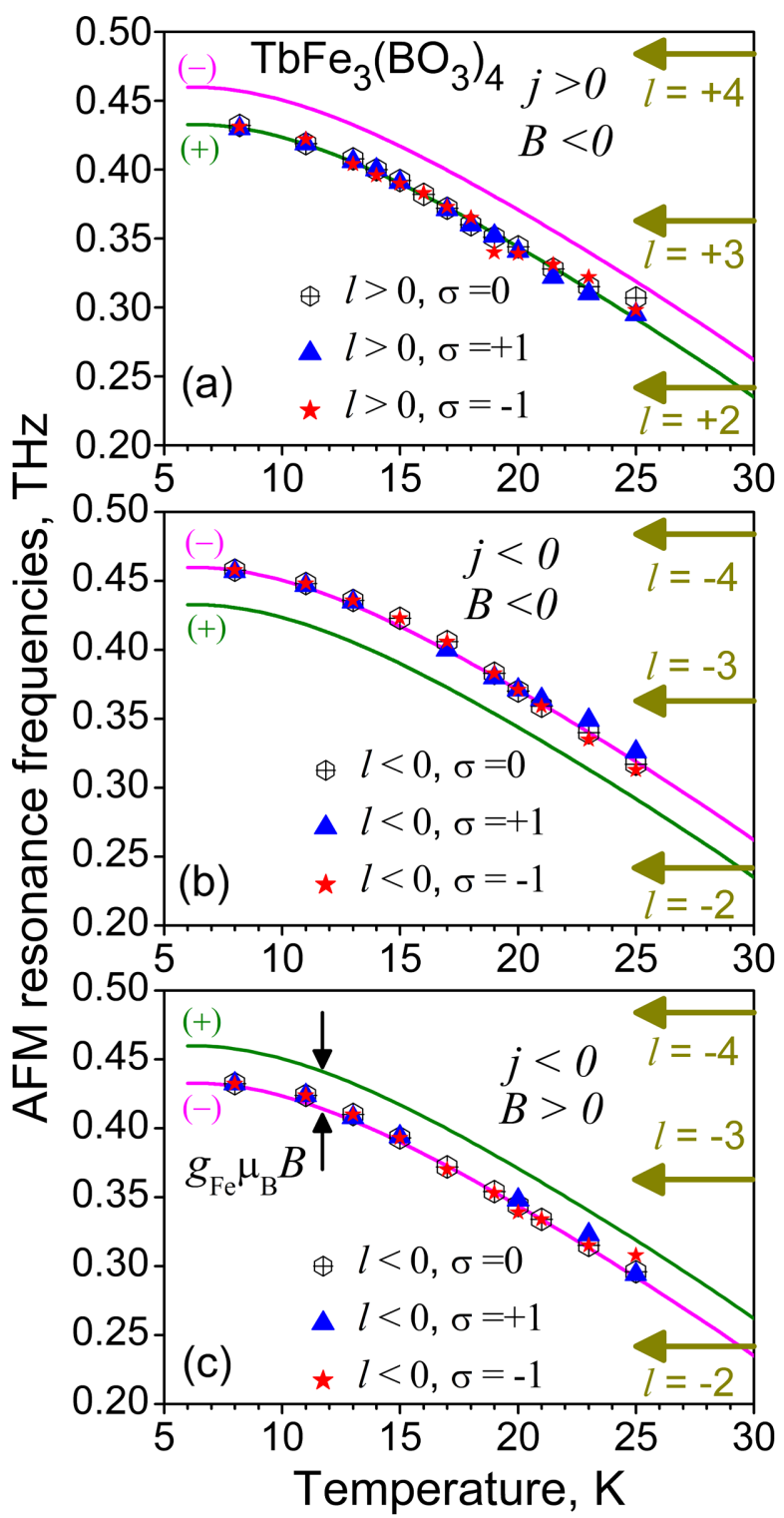

FIG. 4. Total angular momentum dichroism in Tb FB revealed in the temperature dependencies of the AFM resonance frequencies $\hbar \Omega_{M}^{ \pm}(B, T)$ for different combinations of the $\vec{B}$ direction and the sign of $j$ for the vortex beams. (a) $j>0, B<0$, (b) $j<0$, $B<0$, and (c) $j<0, B>0$. Solid curves correspond to the AFM frequencies $\hbar \Omega_{M}^{ \pm}(B, T)$ measured using conventional circularly polarized light. The color code for the curves (olive and magenta) is the same as for $\hbar \Omega_{M}^{ \pm}(B)$ lines in Fig. 1(e). The measurement geometry is the same as shown in Fig. 2(c) but with various input polarizations $\vec{e}_{y}$ for black, $\vec{e}_{L}$ for blue, and $\vec{e}_{R}$ for red symbols.

magnetic ions of $\mathrm{Ni}^{2+}$ and $\mathrm{Fe}^{3+}$ seem not to be important for the vortex beam absorption at the magnon frequencies. Thus, we predict a similar total AM dichroism for many AFM systems. The observed coupling between terahertz beams with high orders of the total AM with the ordered spin structure may provide an impetus for studies of nonlocal collective spin and carrier excitations in other quantum systems, such as Landau levels in 2D electron gas 
and graphene, chiral modes in Weyl and Dirac semimetals, and $d$-wave superconductors.

The authors are grateful to K. Watt for the design of the spiral plates, to V. Kiryukhin for useful discussions, and to I. A. Gudim for the Tb FB crystal. Work at the New Jersey Institute of Technology and Rutgers was supported by the U.S. DOE under Contract No. DEFG02-07ER46382. The work at the University of Fribourg was funded by the Schweizerische Nationalfonds (SNF) by Grant No. 200020-172611. A. A. S. is grateful to SNF for the IZSEZ0-184564 support during his research visit at the University of Fribourg.

*Corresponding author. sirenko@njit.edu

[1] L. Allen, M. W. Beijersbergen, R. J. C. Spreeuw, and J. P. Woerdman, Phys. Rev. A 45, 8185 (1992).

[2] M. Z. Hasan and C. L. Kane, Rev. Mod. Phys. 82, 3045 (2010).

[3] S.-W. Cheong and M. Mostovoy, Nat. Mater. 6, 13 (2007).

[4] S.-W. Cheong, D. Talbayev, V. Kiryukhin, and A. Saxena, npj Quantum Mater. 3, 19 (2018).

[5] W. Koshibae and N. Nagaosa, Nat. Commun. 7, 10542 (2016).

[6] C. T. Schmiegelow, J. Schulz, H. Kaufmann, T. Ruster, U. G. Poschinger, and F. Schmidt-Kaler, Nat. Commun. 7, 12998 (2016).

[7] A. A. Sirenko, P. Marsik, C. Bernhard, T. N. Stanislavchuk, V. Kiryukhin, and S-W. Cheong, Phys. Rev. Lett. 122, 237401 (2019).

[8] K. A. Forbes and D. L. Andrews, Phys. Rev. Research 1, 033080 (2019).

[9] C. Ritter, A. Balaev, A. Vorotynov, G. Petrakovskii, D. Velikanov, V. Temerov, and I. Gudim, J. Phys. Condens. Matter 19, 196227 (2007).

[10] M. N. Popova, T. N. Stanislavchuk, B. Z. Malkin, and L. N. Bezmaternykh, J. Phys. Condens. Matter 24, 196002 (2012).

[11] I. Zivkovic, K. Prsa, O. Zaharko, and H. Berger, J. Phys. Condens. Matter 22, 056002 (2010).
[12] R. E. Newnham and E. P. Meagher, Mater. Res. Bull. 2, 549 (1967).

[13] R. Becker and H. Berger, Acta Crystallogr. Sect. E 62, i222 (2006).

[14] S. Skiadopoulou, F. Borodavka, C. Kadlec, F. Kadlec, M. Retuerto, Zh. Deng, M. Greenblatt, and S. Kamba, Phys. Rev. B 95, 184435 (2017).

[15] J. C. Joubert, W. B. White, and R. J. Roy, J. Appl. Crystallogr. 1, 318 (1968).

[16] D. Fausti, A. A. Nugroho, P. H. M. van Loosdrecht, S. A. Klimin, M. N. Popova, and L. N. Bezmaternykh, Phys. Rev. B 74, 024403 (2006).

[17] M. N. Popova, E. P. Chukalina, T. N. Stanislavchuk, and L. N. Bezmaternykh, J. Magn. Magn. Mater. 300, e440 (2006).

[18] A. B. Kuz'menko, A. A. Mukhin, V. Yu. Ivanov, A. M. Kadomtseva, S. P. Lebedev, and L. N. Bezmaternykh, Zh. Eksp. Teor. Fiz. 140, 131 (2011) [J. Exp. Theor. Phys. 113, 113 (2011)].

[19] D. Szaller, V. Kocsis, S. Bordács, T. Fehér, T. Rõõm, U. Nagel, H. Engelkamp, K. Ohgushi, and I. Kézsmárki, Phys. Rev. B 95, 024427 (2017).

[20] M. N. Popova, K. N. Boldyrev, S. A. Klimin, T. N. Stanislavchuk, A. A. Sirenko, and L. N. Bezmaternykh, J. Magn. Magn. Mater. 383, 250 (2015).

[21] K. N. Boldyrev, T. N. Stanislavchuk, A. A. Sirenko, D. Kamenskyi, L. N. Bezmaternykh, and M. N. Popova, Phys. Rev. Lett. 118, 167203 (2017).

[22] P. Marsik, K. Sen, J. Khmaladze, M. Yazdi-Rizi, B. P. P. Mallett, and C. Bernhard, Appl. Phys. Lett. 108, 052901 (2016).

[23] http://www.tydexoptics.com/products/thz_optics/thz_lens/ \#thz_axi.

[24] J. Sun, J. Zeng, X. Wang, A. N. Cartwright, and N. M. Litchinitser, Sci. Rep. 4, 4093 (2014).

[25] C. Liu, J. Liu, L. Niu, X. Wei, K. Wang, and Z. Yang, Sci. Rep. 7, 3891 (2017).

[26] See Supplemental Material at http://link.aps.org/ supplemental/10.1103/PhysRevLett.126.157401 for details of the experimental setup and vortex beam characterization.

[27] F. Kepfer and C. Kittel, Phys. Rev. 85, 329 (1952).

[28] T. Oguchi and A. Honma, J. Phys. Soc. Jpn. 16, 79 (1961).

[29] E. I. Rashba and V. I. Sheka, Modern problems in condensed matter sciences 27, 131 (1991). 